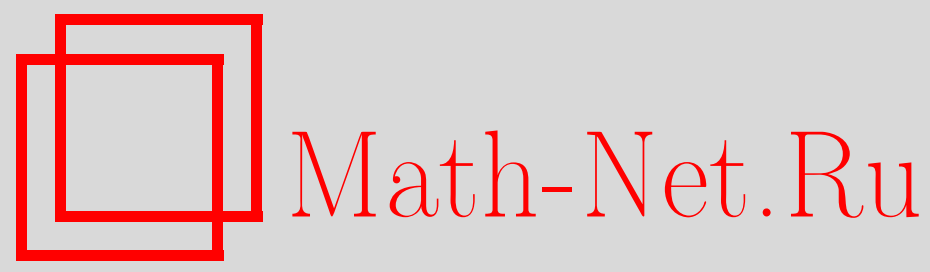

С. Б. Вакарчук, О наилучших линейных методах приближения и поперечниках некоторых классов аналитических функций, Матем. заметки, 1999, том 65, выпуск 2, 186193

DOI: https://doi.org/10.4213/mzm1040

Использование Общероссийского математического портала Math-Net.Ru подразумевает, что вы прочитали и согласны с пользовательским соглашением http://www . mathnet.ru/rus/agreement

Параметры загрузки:

IP : 3.91 .87 .62

26 апреля 2023 г., $17: 29: 21$

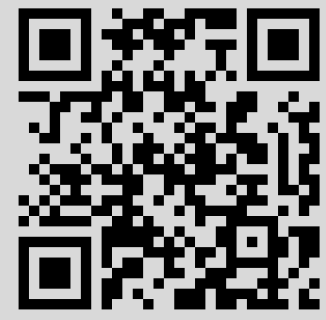




\title{
О НАИЛУЧШИХ ЛИНЕЙНЫХ МЕТОДАХ ПРИБЛИЖЕНИЯ И ПОПЕРЕЧНИКАХ НЕКОТОРЫХ КЛАССОВ АНАЛИТИЧЕСКИХ ФУНКЦИЙ
}

\section{C. Б. Вакарчук}

\begin{abstract}
В пространстве Харди $H_{q}, q \geqslant 1$, указаны наилучшие линейные методы приближения для классов аналитических функций, изучавшихся Н. Айнуллоевым и являющихся в определенном смысле обобщением введенных Л. В. Тайковым функциональных множеств. Найдены точные значения их линейных и гельфандовских $n$-поперечников. Также получены точные значения колмогоровского и бернштейновского $n$-поперечников классов аналитических в $|z|<1$ функций, $K$-функционалы граничных значений которых мажорируются заданной функцией.
\end{abstract}

Библиография: 12 названий.

1. Задача построения наилучших линейных методов приближения для классов аналитических функций и вычисления точньх значений $n$-поперечников вызывает определенный интерес, хотя по данной тематике в настоящее время уже получен целый ряд окончательных результатов (см., например, [1]-[6] и список литературы к ним). В [4] были построены наилучшие линейные методы приближения для некоторых из рассмотренных Л.В. Тайковым [5] функциональных классов. В данной работе указаны наилучшие линейные методы приближения для ряда классов, изучавшихся Н. Айнуллоевьг [6] и являющихся в определенном смысле обобщением функциональных множеств из [5], и найдены точные значения их линейных и гельфандовских $n$-поперечников в пространствах Харди $H_{q}, q \geqslant 1$. Также получены точные значения колмогоровского и бернштейновского $n$-поперечников классов аналитических в единичном круге функций, $K$-функционалы граничных значений которых мажорируются заданной функцией.

Напомним, что функция $f(z)$ аналитическая в единичном круге $|z|<1$ принадлежит банахову пространству $H_{q}$, если

$$
\|f\|_{H_{q}}=\lim _{\rho \rightarrow 1-0} M_{q}(f, \rho)<\infty, \quad M_{q}(f, \rho)=\left(\frac{1}{2 \pi} \int_{0}^{2 \pi}\left|f\left(\rho e^{i x}\right)\right|^{q} d x\right)^{1 / q} .
$$

При этом норма функции $f(z) \in H_{q}$ реализуется на ее угловых граничных значениях $f\left(e^{i x}\right)$. Обозначим через $H_{q, \rho}, 0<\rho \leqslant 1$, пространство Харди аналитических в круге $|z|<\rho$ функций $f(z)$, для которых $\|f(z)\|_{H_{q, \rho}}=\|f(\rho z)\|_{H_{q}}<\infty$. Производную 
$r$-го порядка функции $f(z)$ по аргументу $x$ комплексного переменного $z=\rho e^{i x}$ обозначим через $f_{a}^{(r)}(z)$. Очевидно, что

$$
\begin{gathered}
f_{a}^{(1)}(z)=\frac{\partial f(z)}{\partial x}=f_{a}^{(1)}(z), \quad z_{a}^{(1)}=f_{a}^{(1)}(z) z i \\
f_{a}^{(r)}(z)=\left(f_{a}^{(r-1)}(z)\right)_{a}^{(1)}, \quad r=2,3, \ldots
\end{gathered}
$$

Если $f_{a}^{(r)}(z) \in H_{q}$, то структурные свойства этой функции можно либо охарактеризовать быстротой стремления к 0 модуля гладкости ее граничных значений

$$
\omega_{2}(f, 2 x)_{H_{q}}=\sup _{|\tau| \leqslant x}\left\{\left\|f_{a}^{(r)}\left(e^{i(\cdot+\tau)}\right)-2 f_{a}^{(r)}\left(e^{i(\cdot)}\right)+f_{a}^{(r)}\left(e^{i(\cdot-\tau)}\right)\right\|_{H_{q}}\right\}
$$

при $x \rightarrow 0$, либо задать скорость убьвания к 0 мажоранты некоторой усредненной величины, содержащей $\omega_{2}(f, 2 x)_{H_{q}}[5],[6]$.

Пусть $\Phi(x), x \geqslant 0,-$ произвольная возрастающая непрерывная функция, $\Phi(0)=0$. Для любого заданного значения параметра $\mu \geqslant 1 / 2$ обозначим через $W_{a}^{r} H_{q}(\Phi, \mu), r \in \mathbb{N}$, класс функций $f(z) \in H_{q}$, для которых производные $f_{a}^{(r)}(z) \in H_{q}$ удовлетворяют условию

$$
\frac{1}{t} \int_{0}^{t} \omega_{2}\left(f_{a}^{(r)}, 2 x\right)_{H_{q}}\left(1+\left(\mu^{2}-1\right) \sin \frac{\pi x}{2 t}\right) d x \leqslant \Phi(t)
$$

для любого $t \in(0, \pi / 2]$.

Обозначим через $d_{n}(\mathfrak{M}, X), d^{n}(\mathfrak{M}, X), b_{n}(\mathfrak{M}, X), \delta_{n}(\mathfrak{M}, X)$ соответственно колмогоровский, гельфандовский, бернштейновский и линейньй $n$-поперечники некоторого выпуклого центрально-симметричного компакта $\mathfrak{M}$ в банаховом пространстве $X$ (см., например, [1], [4], [7]). Данные $n$-поперечники связаны следуюшими соотношениями:

$$
b_{n}(\mathfrak{M}, X) \leqslant d_{n}(\mathfrak{M}, X) \leqslant \delta_{n}(\mathfrak{M}, X), \quad b_{n}(\mathfrak{M}, X) \leqslant d^{n}(\mathfrak{M}, X) \leqslant \delta_{n}(\mathfrak{M}, X) .
$$

Из результатов [6] следует, что если при заданном $\mu \geqslant 1 / 2$ и при любых $t, \tau \in(0, \pi / 2]$ функция $\Phi$ удовлетворяет условию

$$
\frac{\pi}{\pi-2} \int_{0}^{1}\left(1-\cos \frac{\pi t x}{2 \tau \mu}\right)_{*}\left(1+\left(\mu^{2}-1\right) \sin \frac{\pi x}{2}\right) d x \leqslant \frac{\Phi(t)}{\Phi(\tau)},
$$

где

$$
(1-\cos x)_{*}= \begin{cases}1-\cos x, & \text { если } 0 \leqslant x \leqslant \pi, \\ 2, & \text { если } x \geqslant \pi,\end{cases}
$$

то для любого натурального числа $n \geqslant 2$ справедливы равенства

$$
b_{n}\left(W_{a}^{r} H_{q}(\Phi, \mu) ; H_{q}\right)=d_{n}\left(W_{a}^{r} H_{q}(\Phi, \mu) ; H_{q}\right)=\frac{\pi}{2(\pi-2) n^{r}} \Phi\left(\frac{\pi}{2 n \mu}\right) .
$$

Там же приведен пример степенной функции $\Phi(t)=t^{\alpha(\mu)}$, где

$$
\alpha(\mu)=\frac{\pi^{2}}{2(\pi-2) \mu} \int_{0}^{1} x\left(1+\left(\mu^{2}-1\right) \sin \frac{\pi x}{2}\right) \sin \frac{\pi x}{2 \mu} d x
$$


которая удовлетворяет условию (2), и отмечено, что случай $\alpha(1)=2 /(\pi-2)$ содержится в $[5]$.

В теории аппроксимации функций часто используется идея замены произвольной функции $f$ достаточно гладкой функцией $g$. Одна из наиболее эффективных реализаций этой идеи основана на методе $K$-функционала Петре теории интерполяционных пространств [8].

В пространстве Харди $H_{q}$ для произвольной функции $f(z) \in H_{q}$ определим $K$-функционал следуюшим образом:

$$
K_{j, q}(f, \nu)=\inf _{g_{a}^{(j)} \in H_{q}}\left\{\|f-g\|_{H_{q}}+\nu^{j}\left\|g_{a}^{(j)}\right\|_{H_{q}}\right\},
$$

где $\nu>0, j \in \mathbb{N}, 1 \leqslant q<\infty$. При $q=\infty$ полагаем, что $f(z)$ имеет непрерывные угловые граничные значения $f(t) \stackrel{\text { df }}{=} f\left(e^{i t}\right)$, т.е. $f(t) \in C[0,2 \pi]$. Из рассуждений [9], [10, с. 60] применительно к функциям $f(z) \in H_{q}$ следует, что если $1 \leqslant q<\infty, j \in \mathbb{N}$ и $0<\nu \leqslant \pi$, TO

$$
\omega_{j}(f, \nu)_{q} \asymp K_{j, q}(f, \nu)
$$

где

$$
\omega_{j}(f, \nu)_{q}=\sup _{0 \leqslant h \leqslant \nu}\left\|\sum_{s=0}^{j}(-1)^{j-s}\left(\begin{array}{l}
i \\
s
\end{array}\right) f\left(e^{i(\cdot+s h)}\right)\right\|_{H_{q}}
$$

- модуль гладкости $j$-го порядка угловых граничных значений функции $f$. Соотношение (5) справедливо и в случае $q=\infty$, если $f(t) \in C[0,2 \pi]$.

Пусть $\mathcal{K}(t)$ - положительная монотонно возрастающая функция при $t>0$,

$$
\lim _{t \rightarrow 0} \mathcal{K}(t)=\mathcal{K}(0)=0 .
$$

Обозначим через $H_{q}(\mathcal{K})$ класс функций $f(z) \in H_{q}$, каждая из которых при некотором $j \in \mathbb{N}$ удовлетворяет условиям

$$
K_{j, q}\left(f, \frac{1}{m}\right) \leqslant \mathcal{K}\left(\frac{1}{m}\right), \quad m \in \mathbb{N} .
$$

Отметим, что в силу (5) такое определение класса оправдано.

Основными результатами данного сообщения являются следующие теоремы.

ТЕорема 1. Если мажсоранта $\Phi(x)$ при некотором заданном $\mu \geqslant 1 / 2$ удовлетворяет условию (2), то для любого натурального числа $n \geqslant 2$ имеют место равенства

$$
d^{n}\left(W_{a}^{r} H_{q}(\Phi, \mu) ; H_{q}\right)=\delta_{n}\left(W_{a}^{r} H_{q}(\Phi, \mu) ; H_{q}\right)=\frac{\pi}{2(\pi-2) n^{r}} \Phi\left(\frac{\pi}{2 n \mu}\right) .
$$

ТЕОрема 2. Пусть махсоранта $\mathcal{K}(t)$ для любьх $m, s \in \mathbb{N}, m \geqslant s$, удовлетворяет неравенству

$$
\frac{\mathcal{K}(1 / m)}{\mathcal{K}(1 / s)} \geqslant \frac{s}{m}
$$

Тогда для произвольного $n \in \mathbb{N} u 0<\rho<1$ справедливы равенства

$$
d_{n}\left(H_{q}(\mathcal{K}) ; H_{q, \rho}\right)=b_{n}\left(H_{q}(\mathcal{K}) ; H_{q, \rho}\right)=\rho^{n} \mathcal{K}\left(\frac{1}{n}\right)
$$


2. Доказательство теоремы 1. Построим наилучший линейньй метод приближения функций из класса $W_{a}^{r} H_{q}(\Phi, \mu)$ в пространстве $H_{q}$. Для произвольной функции

$$
f(z)=\sum_{k=0}^{\infty} c_{k} z^{k}
$$

принадлежащей $W_{a}^{r} H_{q}(\Phi, \mu)$, запишем следующее соотношение:

$$
f(z)-\mathfrak{L}_{n-1, r}(f, z)=\frac{1}{2 \pi i^{r}} \int_{0}^{2 \pi} f_{a}^{(r)}\left(z e^{-i x}\right) e^{i n x}\left(n^{-r}+2 \sum_{k=n+1}^{\infty} k^{-r} \cos (k-n) x\right) d x
$$

где

$$
\mathfrak{L}_{n-1, r}(f, z) \stackrel{\mathrm{df}}{=} \sum_{k=0}^{n-1} \lambda_{k, r} c_{k} z^{k}, \quad \lambda_{k, r}= \begin{cases}1, & \begin{array}{l}
\text { если } k=0, \\
1-\left(\frac{k}{2 n-k}\right)^{r},
\end{array} \text { если } k=1, \ldots, n-1 .\end{cases}
$$

Чтобы убедиться в справедливости $(8)$, необходимо разложить $f_{a}^{(r)}(\cdot)$ в ряд Тейлора и затем почленно проинтегрировать.

При решении в [6] ряда экстремальных задач на основе разработанного в [5] подхода важную роль играет аналитическая функция

$$
F_{t}(f, z)=\frac{\pi}{2 t(\pi-2)} \int_{0}^{t}\left(f\left(z e^{i x}\right)+f\left(z e^{-i x}\right)\right)\left(1-\sin \frac{\pi x}{2 t}\right) d x
$$

используемая в процессе промежуточного приближения.

Полагая в (10) $t=t_{*}=\pi /(2 \mu n)$, получим

$$
\begin{aligned}
F_{*}(f, z) & \stackrel{\mathrm{df}}{=} F_{t_{*}}(f, z)=\frac{\mu n}{\pi-2} \int_{0}^{\pi /(2 \mu n)}\left(f\left(z e^{i x}\right)+f\left(z e^{-i x}\right)\right)(1-\sin \mu n x) d x \\
& =\sum_{k=0}^{\infty} \psi_{k, n} c_{k} z^{k}
\end{aligned}
$$

где

$$
\psi_{k, n}=\frac{2 \mu n}{\pi-2} \int_{0}^{\pi /(2 \mu n)} \cos k x(1-\sin \mu n x) d x .
$$

Очевидно, что $F_{*}(f, z)$ принадлежит $H_{q}$, если $f(z)$ является элементом этого пространства.

Запишем для $f_{a}^{(r)}(z)$ функцию

$$
F_{*}\left(f_{a}^{(r)}, z\right)=\sum_{k=1}^{\infty} \psi_{k, n}(i k)^{r} c_{k} z^{k}
$$


и построим следуюший вспомогательный оператор:

$$
\begin{aligned}
\Lambda_{n-1, r}(f, z) \stackrel{\text { df }}{=} & \frac{1}{2 \pi i^{r}} \int_{0}^{2 \pi} \mathfrak{L}_{n-1,2}\left(F_{*}\left(f_{a}^{(r)}\right), z e^{-i x}\right) \\
& \times e^{i n x}\left(n^{-r}+2 \sum_{k=n+1}^{\infty} k^{-r} \cos (k-n) x\right) d x \\
= & \sum_{k=1}^{n-1}\left(1-\left(\frac{k}{2 n-k}\right)^{2}\right)\left(\frac{k}{2 n-k}\right)^{r} \psi_{k, n} c_{k} z^{k}
\end{aligned}
$$

Поскольку последовательность $\left\{(n+k)^{-r}\right\}_{k=0}^{\infty}$ является выпуклой, ряд

$$
\frac{1}{2 n^{r}}+\sum_{k=n+1}^{\infty} k^{-r} \cos (k-n) x
$$

сходится всюду, за исключением быть может точки $x=0$, к некоторой неотрицательной интегрируемой функции $g(x)$ и является рядом Фурье этой функции (см., например, [11]). Используя этот факт, соотношение (8), неравенство Минковского и полагая $\Pi_{n-1, r}(f, z)=\mathfrak{L}_{n-1, r}(f, z)+\Lambda_{n-1, r}(f, z)$, имеем

$$
\left\|f-\Pi_{n-1, r}(f)\right\|_{H_{q}} \leqslant n^{-r}\left(\left\|f_{a}^{(r)}-F_{*}\left(f_{a}^{(r)}\right)\right\|_{H_{q}}+\left\|F_{*}\left(f_{a}^{(r)}\right)-\mathfrak{L}_{n-1,2}\left(F_{*}\left(f_{a}^{(r)}\right)\right)\right\|_{H_{q}}\right) .
$$

Оценку сверху первого слагаемого в правой части неравенства (11) получаем сразу же, применив неравенство Минковского и определение величины $\omega_{2}(f, 2 x)_{H_{q}}$ :

$$
\left\|f_{a}^{(r)}-F_{*}\left(f_{a}^{(r)}\right)\right\|_{H_{q}} \leqslant \frac{\mu n}{\pi-2} \int_{0}^{\pi /(2 \mu n)} \omega_{2}\left(f_{a}^{(r)}, 2 x\right)_{H_{q}}(1-\sin \mu n x) d x .
$$

Прежде чем приступить к оценке второго слагаемого в правой части $(12)$, как и в [5, c. 289], отметим, что $f(z)$ можно считать произвольным полиномом $p_{m}(z)$ любого порядка $m$. Поскольку множество всех полиномов всюду плотно в $H_{q}$, осуществляемые далее математические операции над функцией $f(z)$ являются корректными. Используя (8), запишем

$$
\left\|F_{*}\left(f_{a}^{(r)}\right)-\mathfrak{L}_{n-1,2}\left(F_{*}\left(f_{a}^{(r)}\right)\right)\right\|_{H_{q}} \leqslant n^{-2}\left\|\left(F_{*}\left(f_{a}^{(r)}\right)\right)_{a}^{(2)}\right\|_{H_{q}}=n^{-2}\left\|F_{*}\left(f_{a}^{(r+2)}\right)\right\|_{H_{q}} .
$$

Воспользовавшись далее определением функции $F_{*}(\cdot)$ и дважды интегрируя по частям, на основании неравенства Минковского получим следующее продолжение оценки (13):

$$
\left\|F_{*}\left(f_{a}^{(r)}\right)-\mathfrak{L}_{n-1,2}\left(F_{*}\left(f_{a}^{(r)}\right)\right)\right\|_{H_{q}} \leqslant \frac{\mu n}{\pi-2} \int_{0}^{\pi /(2 \mu n)} \omega_{2}\left(f_{a}^{(r)}, 2 x\right)_{H_{q}} \mu^{2} \sin \mu n x d x .
$$

В силу (12)-(14) для любой функции $f(z) \in W_{a}^{r} H_{q}(\Phi, \mu)$ из (11) имеем

$$
\begin{aligned}
\left\|f-\Pi_{n-1, r}(f)\right\|_{H_{q}} & \leqslant \frac{\mu n}{(\pi-2) n^{r}} \int_{0}^{\pi /(2 \mu n)} \omega_{2}\left(f_{a}^{(r)}, 2 x\right)_{H_{q}}\left(1+\left(\mu^{2}-1\right) \sin \mu n x\right) d x \\
& \leqslant \frac{\pi}{2(\pi-2) n^{r}} \Phi\left(\frac{\pi}{2 \mu n}\right)
\end{aligned}
$$


Используя определение линейного $n$-поперечника

$$
\delta_{n}\left(W_{a}^{r} H_{q}(\Phi, \mu) ; H_{q}\right)=\inf _{L_{n} \subset H_{q} \Lambda: H_{q} \rightarrow L_{n}} \operatorname{inp}_{f \in W_{a}^{r} H_{q}(\Phi, \mu)}\|f-\Lambda f\|_{H_{q}},
$$

где $L_{n}$ - линейное $n$-мерное подпространство, а $\Lambda: H_{q} \rightarrow L_{n}$ есть линейный непрерьвный оператор, из (1), (3) и (15) получим

$$
\begin{aligned}
d^{n}\left(W_{a}^{r} H_{q}(\Phi, \mu) ; H_{q}\right) & =\delta_{n}\left(W_{a}^{r} H_{q}(\Phi, \mu) ; H_{q}\right)=\sup _{f \in W_{a}^{r} H_{q}(\Phi, \mu)}\left\|f-\Pi_{n-1, r}(f)\right\|_{H_{q}} \\
& =\frac{\pi}{2(\pi-2) n^{r}} \Phi\left(\frac{\pi}{2 \mu n}\right) .
\end{aligned}
$$

Отсюда следует, что

$$
\Pi_{n-1, r}(f, z)=c_{0}+\sum_{k=1}^{n-1}\left(1+\left(\frac{k}{2 n-k}\right)^{r}\left(\psi_{k, n}\left(1-\left(\frac{k}{2 n-k}\right)^{2}\right)-1\right)\right) c_{k} z^{k}
$$

является наилучшим линейным методом приближения функций из класса $W_{a}^{r} H_{q}(\Phi, \mu)$ в метрике пространства $H_{q}$. Теорема 1 доказана.

3. Доказательство теоремы 2. Для функции $f(z) \in H_{q}(\mathcal{K})$ при $|z|<1$ и $0<\rho<1$ воспользуемся следуюшим представлением:

$$
f(\rho z)-V_{n-1}^{\rho}(f, \rho z)=\frac{\rho^{n}}{2 \pi} \int_{0}^{2 \pi} f\left(z e^{-i x}\right) e^{i n x}\left(1+2 \sum_{k=1}^{\infty} \rho^{k} \cos k x\right) d x
$$

где

$$
V_{n-1}^{\rho}(f, z) \stackrel{\mathrm{df}}{=} \sum_{k=0}^{n-1}\left(1-\rho^{2(n-k)}\right) c_{k} z^{k} .
$$

Чтобы убедиться в справедливости (16), необходимо разложить подынтегральную функцию $f(\cdot)$ в ряд Тейлора и затем почленно проинтегрировать.

Используя введенньй в п. 2 полиномиальный оператор (9) при $r=j$, для произвольной аналитической в $|z|<1$ функции

$$
g(z)=\sum_{k=0}^{\infty} \beta_{k} z^{k}
$$

у которой $g_{a}^{(j)}(z) \in H_{q}$, рассмотрим оператор

$$
Q_{n-1, j}^{\rho}(g, \rho z)=\frac{\rho^{n}}{2 \pi} \int_{0}^{2 \pi} \mathfrak{L}_{n-1, j}\left(g, z e^{-i x}\right) e^{i n x}\left(1+2 \sum_{k=1}^{\infty} \rho^{k} \cos k x\right) d x .
$$

Легко проверить, что

$$
Q_{n-1, j}^{\rho}(g, \rho z)=\beta_{0} \rho^{2 n}+\sum_{k=1}^{n-1}\left(1-\left(\frac{k}{2 n-k}\right)^{j}\right) \rho^{2(n-k)} \beta_{k} z^{k} .
$$


Так как числовая последовательность $\left\{\rho^{k}\right\}_{k=0}^{\infty}$ является вьпуклой, то, рассуждая как и при выводе оценки (11), из (16), (17) имеем

$$
\left\|f(\rho z)-V_{n-1}^{\rho}(f, \rho z)-Q_{n-1, j}^{\rho}(g, \rho z)\right\|_{H_{q}} \leqslant \rho^{n}\left(\|f-g\|_{H_{q}}+\left\|g-\mathfrak{L}_{n-1, j}(g)\right\|_{H_{q}}\right) .
$$

Полагая, в (8) $f \equiv g, r=j$, продолжим данное неравенство следующим образом:

$$
\left\|f(\rho z)-V_{n-1}^{\rho}(f, \rho z)-Q_{n-1, j}^{\rho}(g, \rho z)\right\|_{H_{q}} \leqslant \rho^{n}\left(\|f-g\|_{H_{q}}+n^{-j}\left\|g_{a}^{(j)}\right\|_{H_{q}}\right) .
$$

Из формулы (4) и неравенств (18), (19) следует оценка сверху для наилучшего приближения функции $f(z) \in H_{q}(\mathcal{K})$ подпространством $\mathcal{P}_{n-1}$ алгебраических полиномов степени $\leqslant n-1$ в круге $|z|<\rho$ :

$$
E_{n-1}\left(f ; H_{q, \rho}\right) \stackrel{\mathrm{df}}{=} \inf _{p_{n-1} \in \mathcal{P}_{n-1}}\left\|f-p_{n-1}\right\|_{H_{q, \rho}} \leqslant \rho^{n} K_{j, q}\left(f, \frac{1}{n}\right) .
$$

На основании определений класса $H_{q}(\mathcal{K})$ и колмогоровского $n$-поперечника получим оценку сверху:

$$
d_{n}\left(H_{q}(\mathcal{K}) ; H_{q, \rho}\right)=\inf _{L_{n} \subset H_{q, \rho}} \sup _{f \in H_{q}(\mathcal{K})} \inf _{\varphi \in L_{n}}\|f-\varphi\|_{H_{q, \rho}} \leqslant \rho^{n} \mathcal{K}\left(\frac{1}{n}\right) .
$$

Для получения оценки снизу бернштейновского $n$-поперечника

$$
b_{n}\left(H_{q}(\mathcal{K}) ; H_{q, \rho}\right)=\sup _{L_{n+1} \subset H_{q, \rho}} \sup _{\varepsilon>0}\left\{\varepsilon B \cap L_{n+1} \subset H_{q}(\mathcal{K})\right\}
$$

где $B$ - шар единичного радиуса в $H_{q, \rho}$, рассмотрим множество

$$
B_{*} \stackrel{\mathrm{df}}{=} \rho^{n} \mathcal{K}\left(\frac{1}{n}\right) B \cap \mathcal{P}_{n}=\left\{p_{n} \in \mathcal{P}_{n}:\left\|p_{n}\right\|_{H_{q, \rho}} \leqslant \rho^{n} \mathcal{K}\left(\frac{1}{n}\right)\right\}
$$

и покажем включение $B_{*} \subset H_{q}(\mathcal{K})$. Из (4) для любого полинома $p_{n}(z)$ следуют очевидные неравенства

$$
K_{j, q}\left(p_{n}, \nu\right) \leqslant\left\|p_{n}\right\|_{H_{q}}, \quad K_{j, q}\left(p_{n}, \nu\right) \leqslant \nu^{j}\left\|\left(p_{n}\right)_{a}^{(j)}\right\|_{H_{q}} .
$$

Нам потребуется следующее неравенство:

$$
\left\|p_{n}\right\|_{H_{q}} \leqslant \rho^{-n}\left\|p_{n}\right\|_{H_{q, \rho}}
$$

полученное Е. Хиллом, Г. Сегё, Я. Д. Тамаркиным (см., например, [12]).

Пусть сначала $m \leqslant n$. Тогда на основании $(22),(23)$ для любого полинома $p_{n} \in B_{*}$ имеем

$$
K_{j, q}\left(p_{n}, \frac{1}{m}\right) \leqslant\left\|p_{n}\right\|_{H_{q}} \leqslant \rho^{n}\left\|p_{n}\right\|_{H_{q, \rho}} \leqslant \mathcal{K}\left(\frac{1}{n}\right) \leqslant \mathcal{K}\left(\frac{1}{m}\right) .
$$

При $m \geqslant n$ на основании тех же соображений, неравенства С. Н. Бернштейна [5]

$$
\left\|\left(p_{n}\right)_{a}^{(j)}\right\|_{H_{q}} \leqslant n^{j}\left\|p_{n}\right\|_{H_{q}}
$$


и соотношения (6) получим

$$
\begin{aligned}
K_{j, q}\left(p_{n}, \frac{1}{m}\right) & \leqslant \frac{1}{m^{j}}\left\|\left(p_{n}\right)_{a}^{(j)}\right\|_{H_{q}}\left(\frac{n}{m}\right)^{j}\left\|p_{n}\right\|_{H_{q}} \\
& \leqslant \rho^{-n}\left(\frac{n}{m}\right)^{j}\left\|p_{n}\right\|_{H_{q, \rho}} \leqslant \frac{n}{m} \mathcal{K}\left(\frac{1}{n}\right) \leqslant \mathcal{K}\left(\frac{1}{m}\right) .
\end{aligned}
$$

Таким образом, на основании $(24),(25)$ включение $B_{*} \subset H_{q}(\mathcal{K})$ доказано. Следовательно,

$$
b_{n}\left(H_{q}(\mathcal{K}) ; H_{q, \rho}\right) \geqslant \rho^{n} \mathcal{K}\left(\frac{1}{n}\right) .
$$

Из (21) и (26) следуют равенства (7), чем и завершается доказательство теоремы 2.

Нетрудно убедиться в том, что условию (6) удовлетворяют, например, функции вида $\mathcal{K}(t)=A t^{\alpha}$, где $A=$ const $>0,0<\alpha \leqslant 1$. В заключение заметим, что из (20), (22) и известного равенства $E_{n-1}\left(z^{n} ; H_{q, \rho}\right)=\rho^{n}$ следует одно экстремальное соотношение

$$
\sup _{f \in H_{q}} \frac{E_{n-1}\left(f ; H_{q, \rho}\right)}{K_{j, q}(f, 1 / n)}=\rho^{n}
$$

которое реализуется на функции $z^{n}$.

\section{СПИСОК ЦИТИРОВАННОЙ ЛИТЕРАТУРЫ}

[1] Pinkus A. n-Widths in Approximation Theory. Berlin: Springer, 1985.

[2] Fisher S. D., Stessin M. I. The $n$-width of the unit ball of $H^{q} / /$ J. Approx. Theory. 1991. V. 67. № 3. P. 347-356.

[3] Фарков Ю. А. Поперечники классов Харди и Бергмана в шаре из $\mathbb{C}^{n} / /$ УМН. 1990. Т. 45. № 5. C. 197-198.

[4] Вакарчук С. Б. Наилучшие линейные методы приближения и поперечники классов аналитических в круге функций // Матем. заметки. 1995. Т. 57. №1. С. 30-39.

[5] Тайков А. В. Поперечники некоторых классов аналитических функций // Матем. заметки. 1977. T. 22. № 2. C. 285-295.

[6] Айнуллоев Н. Поперечники классов аналитических функций в единичном круге // Геометрические вопросы теории функций и множеств. Калинин, 1986. С. 91-101.

[7] Тихомиров В.М. Некоторые вопросы теории приближений. М.: Изд-во МГУ, 1976.

[8] Берг Й., Лефстрем Й. Интерполяционные пространства. Введение. М.: Мир, 1980.

[9] Oswald P. On some approximation properties of real Hardy spaces $(0<p \leqslant 1) / /$ J. Approx. Theory. 1984. V. 40. № 1. P. 45-65.

[10] Schumaker L. L. Spline Functions. Basic Theory. New York: Wiley, 1981.

[11] Зигмунд А. Тригонометрические ряды. Т. 1. М.: Мир, 1965.

[12] Шихалиев Н.И. Неравенства типа С. Н. Бернштейна-А. А. Маркова для аналитических функций // Докл. АН АзССР. 1975. Т. 31. № 8. С. 9-14.

Институт геотехнической механики НАН Украины,

Поступило

г. Днепропетровск 